\title{
Two- and Three-Loop Superdirective Receiving Antennas
}

\author{
Elwin W. Seeley \\ Contribution from U.S. Naval Ordnance Laboratory, Corona, California
}

(Received June 11, 1962; revised August 2, 1962)

The characteristics of two- and three-loop superdirective antenna arrays are presented. At VLF, this type of array appears to have many desirable qualities, and the usual detrimental characteristics associated with superdirectivity are less in evidence. It is shown that the beamwidth is narrowest, the front-to-back voltage and power ratios are greatest, and the position of the back lobes and nulls are most invariant when closely spaced loops are used. Inequalities in signals from the individual loops tend to obscure the front and back lobes and limit the proximity of the loops.

\section{List of Symbols}

$E_{\phi}=$ relative voltage received from direction $\phi$ compared to voltage from one loop.

$\phi=$ angle of received signal in the horizontal plane from the vertical plane in which the loops are located.

$\psi=\frac{2 \pi D}{\lambda} \cos \phi$

$D=$ distance between loops

$\lambda=$ free space wavelength

$-\delta=$ phase delay between loops

$\phi_{0}=$ null position

$\phi_{1}=$ position of side lobe maximum

$R_{1}=$ ratio of front lobe to side lobe amplitude

$R_{0}=$ ratio of front lobe to back lobe amplitude

$R_{P}=$ ratio of power collected by the front lobe to that collected by the back lobes

$I=$ current in the loop antenna

$\eta=120 \pi$, intrinsic impedance of space

$A=$ area of loop

$\beta=2 \pi / \lambda$

$\theta=$ angle in the plane of the loop

$r=$ radius of loop

$r_{0}=$ radius of wire used to make loop

$Z_{L}=$ input impedance of loop

$V_{L}=$ loop voltage

$E_{f}=$ free space radiation field.

\section{Introduction}

At very low frequencies (VLF) a large land area is required to obtain high resolution and unidirectional antenna patterns with conventional arrays. It appears that the array can be greatly reduced in size by using the principle of superdirectivity.

Several authors have discussed the factors that limit superdirectivity in practical antennas [Taylor, 1948; Wilmotte, 1948; Riblet, 1948; Yaru, 1951; di Francis, 1956; Stearns, 1961]. It is limited by ohmic losses, narrow bandwidth, and critical tolerances of antenna parameters. Superdirective receiving antennas are realizable at VLF because these limiting factors are less in evidence. There is very little coupling between loops used at VLF so the bandwidth is not 
narrowed and the ohmic losses are not increased when the loops are used in an array. The tolerances of the loop voltages are critical and will be discussed later. In this paper several characteristics of two- and three-loop arrays are derived and presented.

\section{Radiation Pattern Characteristics}

The important characteristies of the patterns of two- and three-loop arrays will be derived. The positions of the side lobes, and nulls, beamwidth, ratio of side and back lobes to front lobe, and ratio of power collected by front lobe to that collected by back lobes will be presented. Some of the pattern characteristics of the two-loop array have been derived and experimentally verified earlier [Friis, 1925]. This paper will be an extension of the work done by Friis.

\subsection{Two-Loop Array}

The pattern of the two-loop array is easily derived by pattern multiplication from the pattern of a loop and that of a two-element isotropic array [Kraus, 1950a]. Consider two identical loops with $(\delta-\pi)$ phase shift between them, oriented in line in a vertical plane such as loops No. 1 and No. 2 in figure 1. If the loops are receiving vertically polarized energy. the radiation pattern in the horizontal plane is given by (18A) in appendix 5.1 which is

$$
E_{\phi}=\left[2 \cos \phi \sin \left(\frac{\psi+\delta}{2}\right)\right] /-\left(\frac{\psi+\delta}{2}\right)
$$
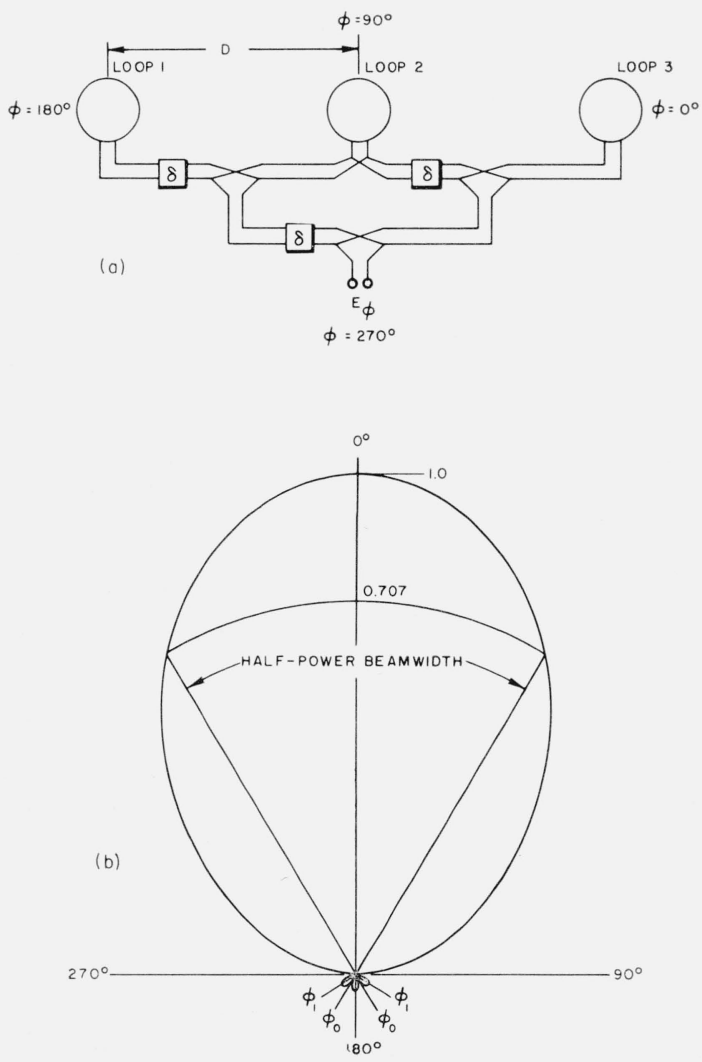

Figure 1. Loop antenna array.

(a) Physical configuration.

(b) Radiation pattern. 


\section{a. Null Positions}

The null positions in the pattern may be used to exclude unwanted signals. There are four nulls: one each at $\phi=90^{\circ}$ and $\phi=270^{\circ}$, and two between the side lobes and the back lobe as shown in figure 1b. Only the null positions between the back lobe and side lobes will be discussed, since the other two are fixed. Null positions between the side lobes were derived in appendix 5.1 and from $(23 \mathrm{~A})$

$$
\phi_{0}=\arccos \left[\frac{-\delta}{\frac{2 \pi D}{\lambda}}\right] .
$$

Equation (2) is plotted in figure 2 which can be used to position the nulls in a loop array.

\section{b. Back Lobe Positions}

A picture of the radiation pattern would not be complete without a knowledge of the side lobes and the back lobe. The side lobes are the two lobes whose peaks are situated at equal angles on either side of $\phi=180^{\circ}$ and the back lobe is the lobe whose peak is at $\phi=180^{\circ}$. If $(22 \mathrm{~A})$ and $(23 \mathrm{~A})$ are substituted into (1) a voltage pattern equation results in terms of the null position selected,

$$
E_{\phi}=2 \cos \phi \sin \left[\frac{\pi D}{\lambda}\left(\cos \phi-\cos \phi_{0}\right)\right] /-\left(\frac{\psi+\delta}{2}\right) .
$$

Only the amplitude is needed,

$$
\left|E_{\phi}\right|=2 \cos \phi \sin \left[\frac{\pi D}{\lambda}\left(\cos \phi-\cos \phi_{0}\right)\right] .
$$

The positions of the maxima of all back lobes can be derived from (4), by differentiating $E_{\phi}$ with respect to $\phi$ and setting the results equal to zero. Two equations are obtained from which three back lobes can be ascertained.

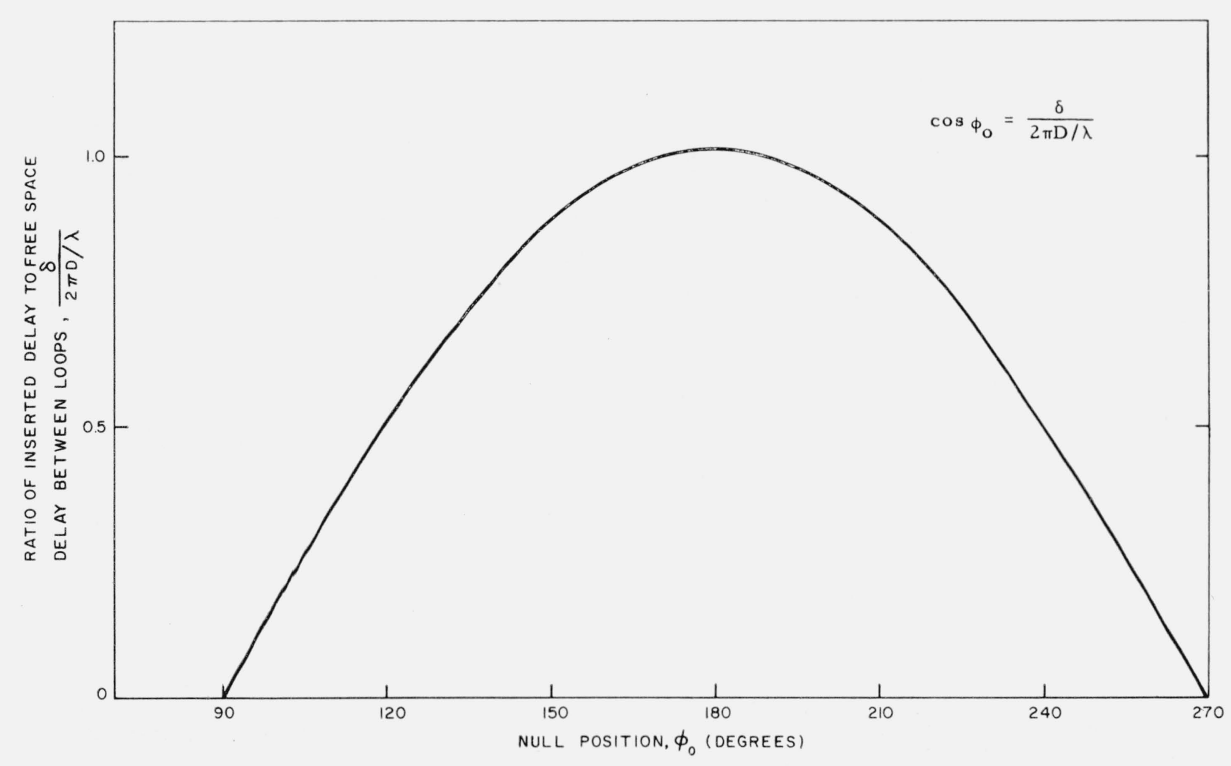

Figure 2. Null position as a function of delay. 
and

$$
\tan \left[\frac{\pi D}{\lambda}\left(\cos \phi_{1}-\cos \phi_{0}\right)\right]=-\frac{\pi D}{\lambda} \cos \phi_{1}
$$

Equation (5) yields one back lobe maximum at $180^{\circ}$, and (6) yields two more side lobe maxima, since there is symmetry about $\phi=0^{\circ}$. A graphical solution of (6) for three different. null positions shows that the side lobe positions change very little with $D / \lambda$. These solutions are plotted in figure 3 . For small loop spacings $(D / \lambda<0.1)$ (6) reduces to

$$
2 \cos \phi_{1}=\cos \phi_{0} .
$$

When the null position has been selected, the corresponding back lobe position may be computed to a fair degree of accuracy especially at VLF frequencies where the wavelength is very long.

\section{c. Beamwidth}

A measure of the directivity of an antenna is its beamwidth. The half-power beamwidth of the two-loop array is determined by setting

$$
E_{\phi A}=0.707 E_{\phi=0}
$$

and solving for $\phi_{A}$, which is half the half-power beamwidth.

$$
\cos \phi_{A} \sin \left[\frac{\pi D}{\lambda}\left(\cos \phi_{A}-\cos \phi_{0}\right)\right]=0.707 \sin \left[\frac{\pi D}{\lambda}\left(1-\cos \phi_{0}\right)\right] .
$$

When (9) is solved and plotted (see fig. 4) the first evidence of superdirectivity is revealed, for the narrowest front lobe occurs with the smallest loop spacing. The beamwidth is only

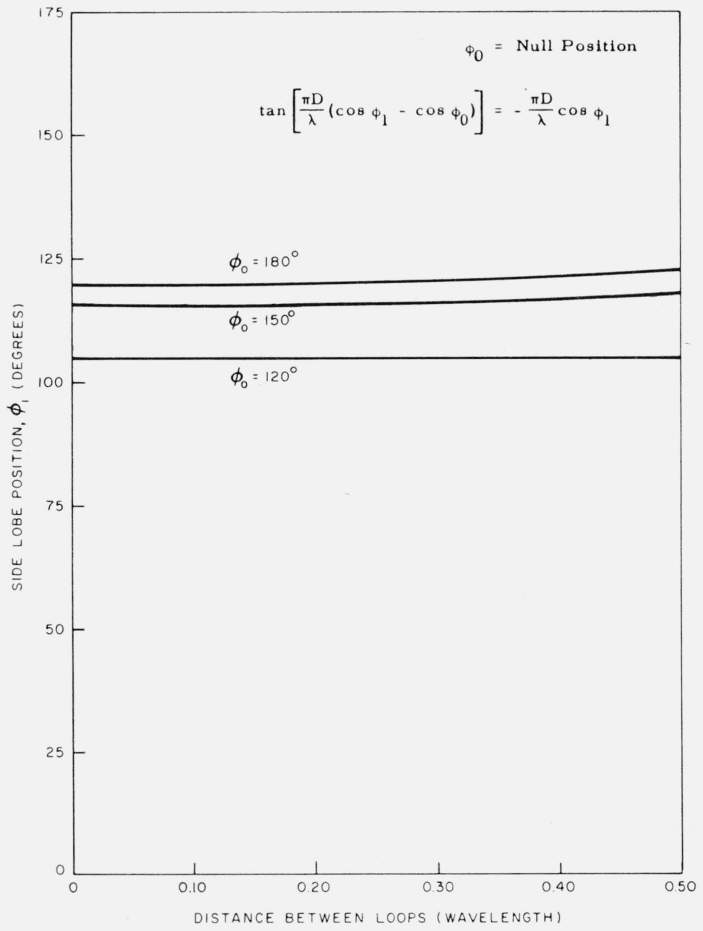

Figure 3. Two-loop array: side-lobe positions.

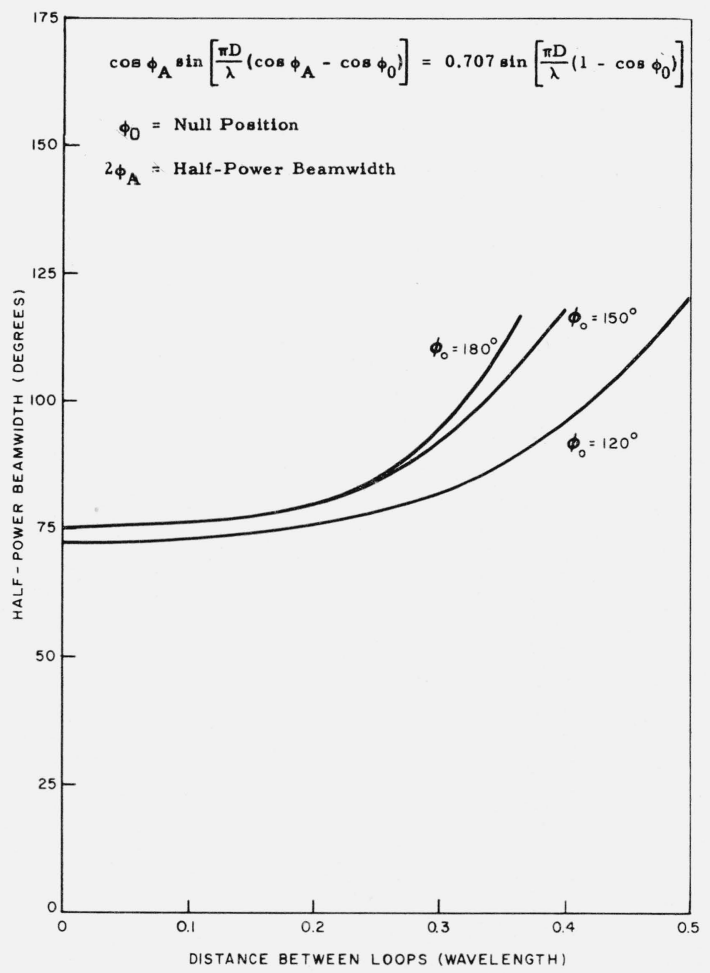

Figure 4. Two-loop array: beamwidth. 
plotted up to a value of loop spacing where lobe splitting begins; at this point the front lobe splits into two lobes. This loop spacing depends upon the null positions, and occurs where

$$
\underset{\phi \rightarrow 0}{\operatorname{Limit}} \frac{d\left|E_{\phi}\right|}{d \phi} \text { is positive. }
$$

Lobe splitting begins at the following loop spacings:

$$
\begin{gathered}
D / \lambda=0.364 \text { for a null at } 180^{\circ} \\
D / \lambda=0.385 \text { for a null at } 150^{\circ} \\
D / \lambda=0.461 \text { for a null at } 120^{\circ} . \\
\text { d. Front-to-Back Lobe Ratios }
\end{gathered}
$$

The front-to-back ratio may well be the most important characteristic of the two-loop array, for it shows to what degree signals from the rear $\left(\phi=90^{\circ}\right.$ to $\left.\phi=270^{\circ}\right)$ are excluded. If (4) with $\phi=0^{\circ}$ is divided by the same equation with $\phi=\phi_{1}$, the position of the back lobe maxima, the lowest front-to-back voltage ratio is obtained for the two lobes on either side of $\phi=180^{\circ}$,

$$
\frac{E_{\phi=0}}{E_{\phi=\phi_{1}}}=R_{1}=\frac{\sin \left[\frac{\pi D}{\lambda}\left(1-\cos \phi_{0}\right)\right]}{\cos \phi_{1} \sin \left[\frac{\pi D}{\lambda}\left(\cos \phi_{1}-\cos \phi_{0}\right)\right]},
$$

and for the single back lobe at $\phi=180^{\circ}$,

$$
\frac{E_{\phi=0}}{E_{\phi=180^{\circ}}}=R_{0}=\frac{\sin \left[\frac{\pi D}{\lambda}\left(1-\cos \phi_{0}\right)\right]}{\sin \left[\frac{\pi D}{\lambda}\left(1+\cos \phi_{0}\right)\right]} .
$$

The front-to-back ratios of back lobes $\phi=\phi_{1}$ and $\phi=180^{\circ}$ are plotted as a function of $D / \lambda$ for several null positions and shown in figures $5 \mathrm{a}$ and $5 \mathrm{~b}$. The two back lobes off $\phi=180^{\circ}$ are called the side lobes to distinguish them from the back lobe at $\phi=180^{\circ}$. These curves represent the lowest front-to-back ratios. At all other angles $\phi$, the front-to-back ratio is greater. It is interesting to note that the solution for $R_{1}$ and $R_{0}$ when $D / \lambda$ is very small is a fair approximation for a good range of $D / \lambda$ values.

$$
\begin{aligned}
& \lim _{\frac{D}{\lambda} \rightarrow 0} R_{1}=\frac{1-\cos \phi_{0}}{\cos \phi_{1}\left(\cos \phi_{1}-\cos \phi_{0}\right)} \\
& \lim _{\frac{D}{\lambda} \rightarrow 0} R_{0}=\frac{1-\cos \phi_{0}}{1+\cos \phi_{0}}
\end{aligned}
$$

When loop spacing is small there is an optimum null position for the greatest front-to-back ratio. If (12) and (13) are plotted as a function of null position, as in figure 6, the intersections of the two curves give the positions of the nulls for equal and optimum front-to-back voltage ratio with all three back lobes. The optimum front-to-back ratio is 11 and occurs at the null positions of 145 and $215^{\circ}$.

In applications where the loop array is in a field of multiple sources, such as spherics at VLF, it is useful to optimize the ratio of the power collected by the front to that collected by the back lobes. For small loop spacings, (4) reduces to

$$
\left|E_{\phi}\right|=\frac{2 \pi D}{\lambda}\left(\cos ^{2} \phi-\cos \phi_{0} \cos \phi\right) .
$$



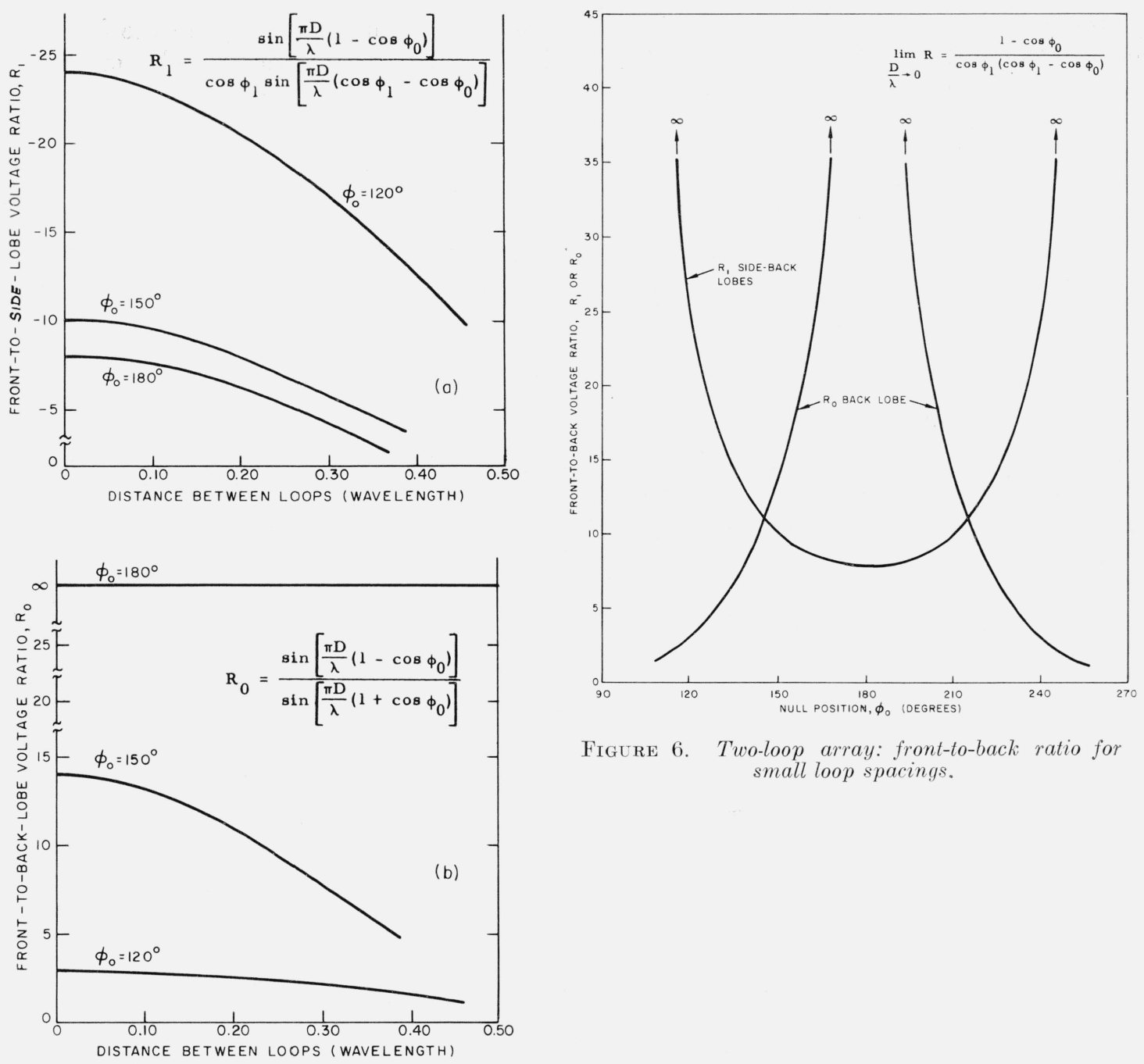

FiguRE 6. Two-loop array: front-to-back ratio for small loop spacings.

Figure 5. Two-loop array.

(a) Front-to-side lobe ratio.

(b) Front-to-back lobe ratio.

If (14) is squared and integrated over the front lobe, the normalized power is obtained.

$$
P_{f}=\frac{8 \pi^{2} D^{2}}{\lambda^{2}} \int_{0}^{\frac{\pi}{2}}\left(\cos ^{2} \phi-\cos \phi_{0} \cos \phi\right)^{2} d \phi
$$

The integration needs to be performed over only half the front lobe ( $\phi=0$ to $\pi / 2)$ since there is symmetry about $\phi=0^{\circ}$. Then

$$
P_{f}=\frac{8 \pi^{2} D^{2}}{\lambda^{2}}\left[\cos \phi \sin \phi\left(\frac{\cos ^{2} \phi}{4}+\frac{3}{8}-\frac{2}{3} \cos \phi_{0} \cos \phi+\frac{\cos ^{2} \phi_{0}}{2}\right)+\phi\left(\frac{\cos ^{2} \phi_{0}}{2}+\frac{3}{8}\right)-\frac{4}{3} \cos \phi_{0} \sin \phi\right]_{0}^{\frac{\pi}{2}},
$$

which reduces to

$$
P_{f}=\frac{8 \pi^{2} D^{2}}{\lambda^{2}}\left(\frac{\pi}{4} \cos ^{2} \phi_{0}-\frac{4}{3} \cos \phi_{0}+\frac{3}{16} \pi\right) .
$$


Likewise, the power in the back lobes is

$$
\begin{aligned}
& P_{b}=\frac{8 \pi^{2} D^{2}}{\lambda^{2}} \int_{\frac{\pi}{2}}^{\pi}\left(\cos ^{2} \phi-\cos \phi_{0} \cos \phi\right)^{2} d \phi, \\
& P_{b}=\frac{8 \pi^{2} D^{2}}{\lambda^{2}}\left(\frac{\pi}{4} \cos ^{2} \phi_{0}+\frac{4}{3} \cos \phi_{0}+\frac{3}{16} \pi\right) .
\end{aligned}
$$

The ratio of power in the front lobe to power in the back lobes is

$$
R_{p}=\frac{\pi \cos ^{2} \phi_{0}-\frac{16}{3} \cos \phi_{0}+\frac{3}{4} \pi}{\pi \cos ^{2} \phi_{0}+\frac{16}{3} \cos \phi_{0}+\frac{3}{4} \pi} .
$$

The front-to-back power ratio depends only on the position of the nulls in the back when loop spacing is small. An optimum null position for maximum front-to-back power ratio is found by differentiating (20), setting the result equal to zero, and solving for $\phi_{0}$. The optimum $\phi_{0}$ equals $150^{\circ} . \quad R_{\max }$ is also found.

$$
\left.R_{\max }\right]_{\phi_{0}=150^{\circ}}=\frac{2 \pi \cos \phi_{0}-\frac{16}{3}}{2 \pi \cos \phi_{0}+\frac{16}{3}}=100 .
$$

It is evident that the best possible ratio of power collected by the front lobe to that collected by the back lobes for the two-loop array with closely spaced loops is 100 . As a comparison, $R_{P}$ is plotted as a function of the null position in figure 7 . The optimum point is clearly seen to be a null position of $150^{\circ}$.

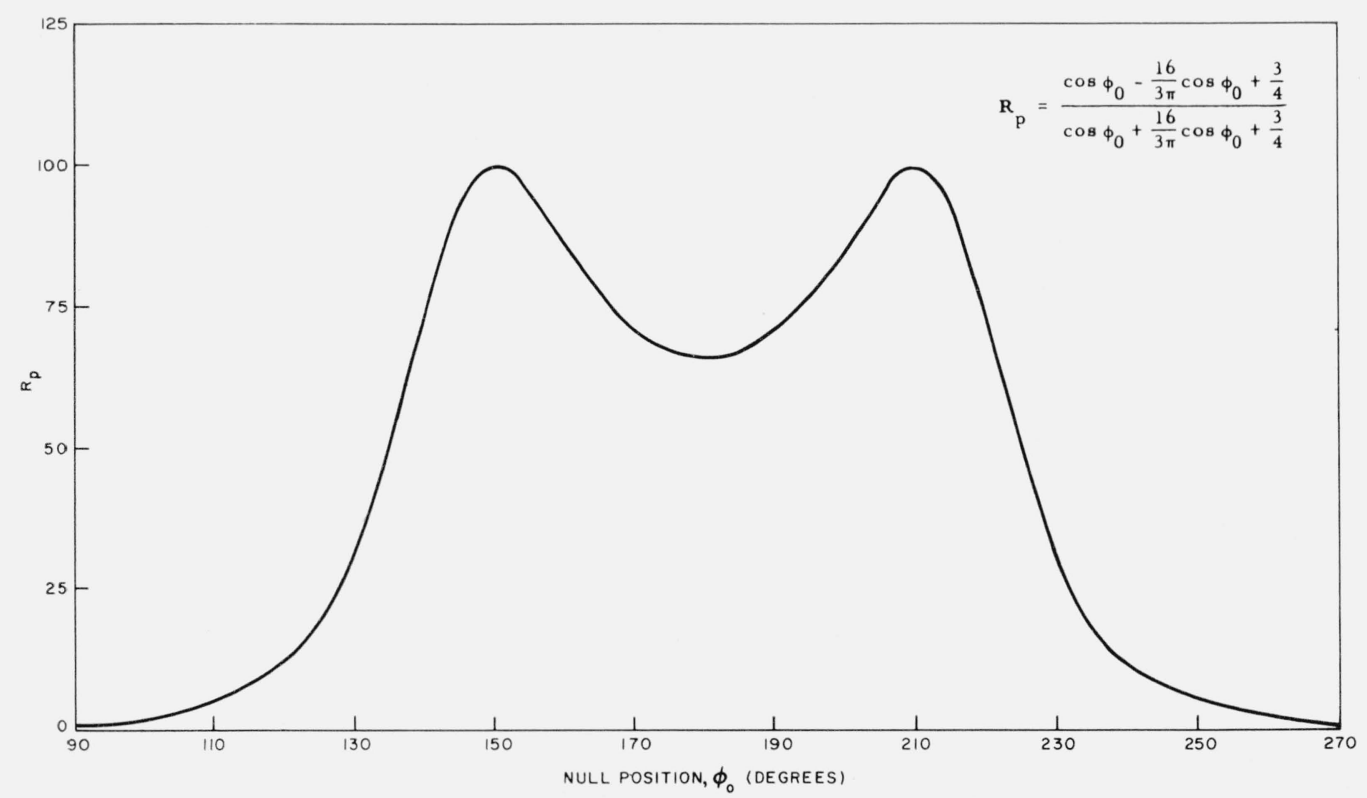

Figure 7. Two-loop array: ratio of power in the front lobe to power in back lobes. 


\subsection{Three-Loop Array}

The pattern of the three-loop array is easily derived by pattern multiplication from the pattern of a loop and that of a three-element isotropic array [Kraus, 1950]. If the three identical loops are oriented in a vertical plane, receiving vertically polarized energy, with $(\delta-\pi)$ phase difference between adjacent loops, as shown in figure $\mathrm{A} 1$, the radiation pattern is given by (5A) from appendix 5.1, and is

$$
E_{\phi}=2 \cos \phi[1-\cos (\psi+\delta)] \underline{l-\delta} .
$$

Equation (22) is the basic equation used to derive the pattern characteristic in the discussion following.

\section{a. Null Positions}

The null positions in the radiation pattern may be used to exclude unwanted signals. Null positions between the side lobes are derived in appendix 5.1. By rearranging (10A) the null position is

$$
\phi_{0}=\arccos \left(\frac{\delta}{2 \pi D / \lambda}\right)
$$

Equation (23) is identical to (2). Apparently the null position is the same for the two- and three-loop arrays when the same delay is used between loops. In figure 2, the null positions are plotted as a function of the ratio of line delay to free space delay between loops. The curve can be used to place the nulls at desired positions in a practical array.

\section{b. Back Lobe Positions}

To gain a clear picture of the radiation pattern, the position of the back lobes including side lobes should be derived. The positions of the back and side lobes are needed also to compute the front-to-back lobe ratio which is a measure of the directivity. If (22) is rearranged in terms of the null position and simplified as in appendix 5.1, the amplitude, which is all that is needed is

$$
\left|E_{\phi}\right|=4 \cos \phi \sin ^{2}\left[\frac{\pi D}{\lambda}\left(\cos \phi-\cos \phi_{0}\right)\right] \text {. }
$$

If (24) is differentiated and set equal to zero, the result is

$$
\sin \phi=0 \quad \phi=0^{\circ} \text { and } 180^{\circ},
$$

which is the maximum of the back lobe, and

$$
-\frac{2 \pi D}{\lambda} \cos \phi_{1}=\sin \left[\frac{\pi D}{\lambda}\left(\cos \phi_{1}-\cos \phi_{0}\right)\right]
$$

which is the equation for the position of the side lobes. Equation (25) has been solved graphically and plotted in figures 8 and 9 . It appears that the distance between the loops has very little effect on the position of the side lobes (fig. 8), but the side lobe positions depend to a great extent on the position of the null (fig. 9). The loop spacing for a VLF superdirective array would be very small compared to a wavelength. In this case (25) reduces to

$$
3 \cos \phi_{1}=\cos \phi_{0} .
$$

The positions of the side lobes, $\phi_{1}$, can readily be computed with (26) when the null position, $\phi_{0}$, has been selected. 
Figure 8. Three-loop array: side lobe positions as a function of loop spacing.
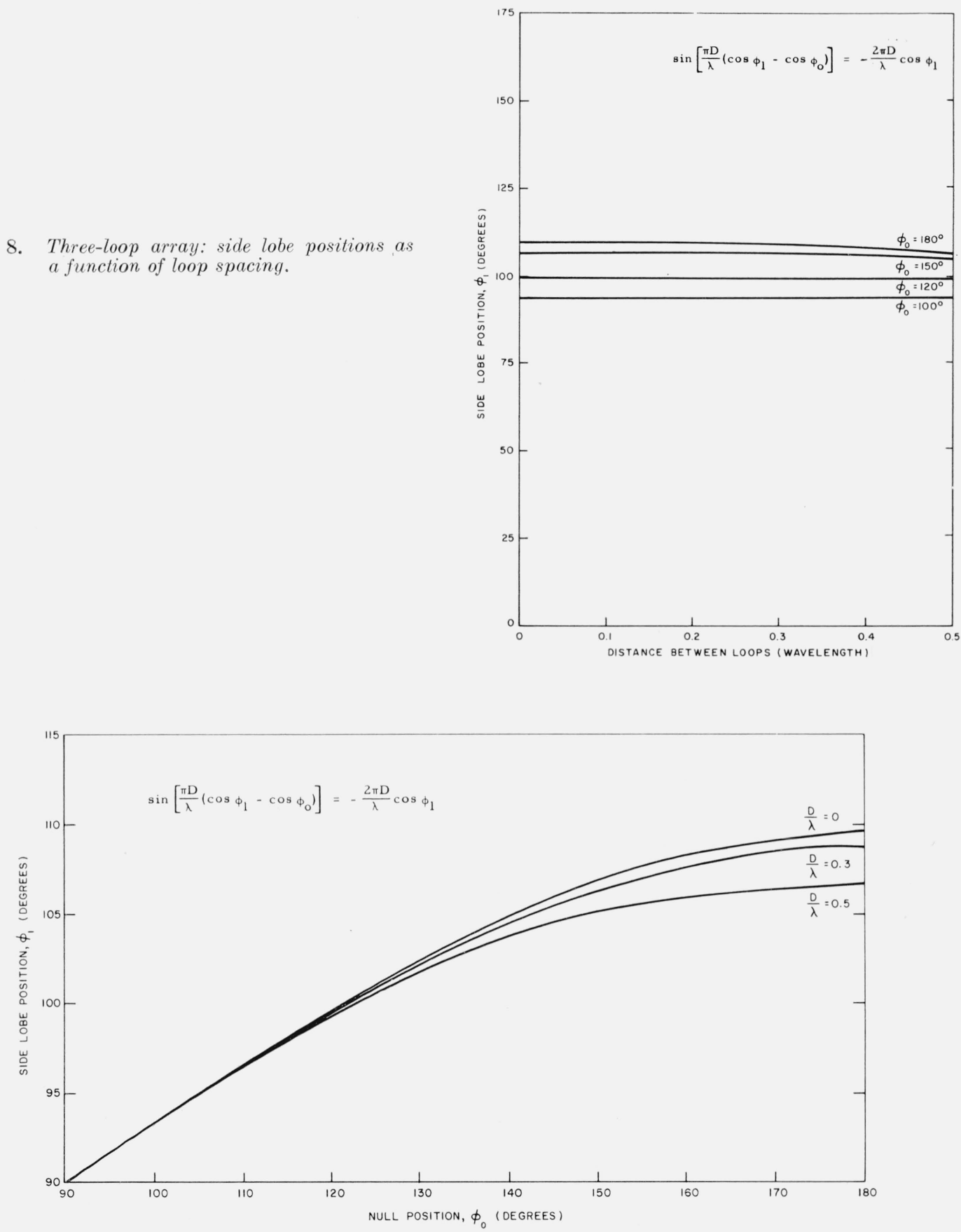

FIGURE 9. Three-loop array: side-lobe positions as a function of null position.

\section{c. Beamwidth}

One measure of the directivity of an antenna is its beamwidth. The half-power beamwidth of the three-loop array is determined by setting $E_{\phi_{A}}=0.707 E_{\phi=0}$, which gives

$$
\cos \phi_{A} \sin ^{2}\left[\frac{\pi D}{\lambda}\left(\cos \phi_{A}-\cos \phi_{0}\right)\right]=0.707 \sin ^{2}\left[\frac{\pi D}{\lambda}\left(1-\cos \phi_{0}\right)\right]
$$

where $2 \phi_{A}$ is the half-power beamwidth. 

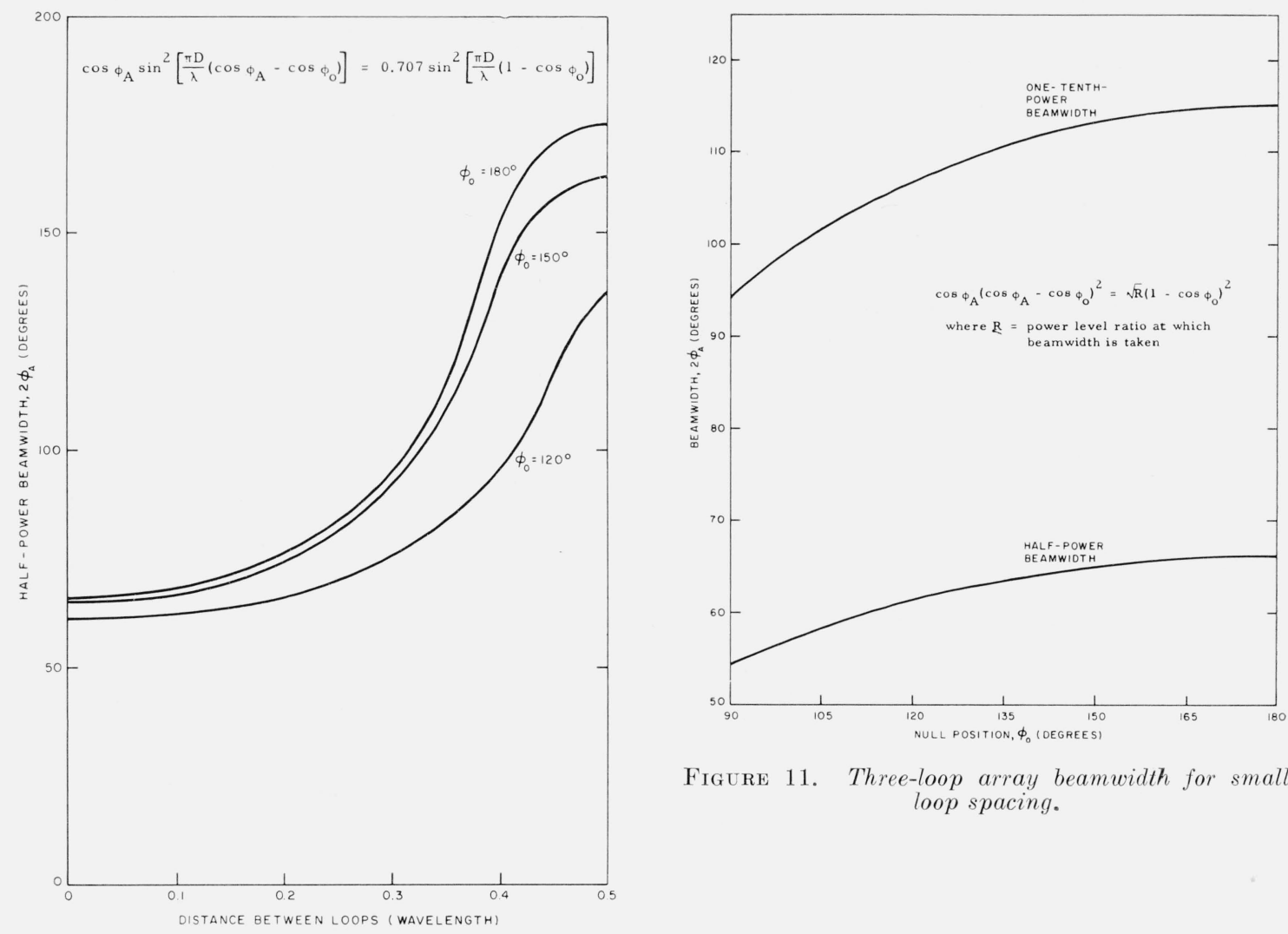

Figure 11. Three-loop array beamwidth for small loop spacing.

Figure 10. Three-loop array beamwidth.

When (27) is solved graphically and plotted (fig. 10), it is readily apparent that the narrowest front lobe and the most directivity occur at the smallest loop spacing. This behavior, which seems contrary to normal array theory, is superdirectivity. At close loop spacing, (27) reduces to

$$
\cos \phi_{A}=\sqrt{R}\left(\frac{1-\cos \phi_{0}}{\cos \phi_{A}-\cos \phi_{0}}\right)^{2}
$$

where $2 \phi_{A}$ is the beamwidth at power level $R$, which is 0.5 for half-power and 0.1 for one-tenthpower beamwidth.

The position of the null has some effect on the beamwidth, as shown in figure 11 . The advantages of the small beamwidth associated with null angles close to $90^{\circ}$ are partially offset by a large back lobe at $\phi=180^{\circ}$. The front lobe is narrowed considerably by using three loops instead of two loops. Figure 12 shows the beamwidth of both two- and three-loop arrays as a function of the null position. These curves can be used to sketch the radiation patterns with selected null positions if the levels of the back lobes are known. One other consideration in sketching the pattern is the loop spacing at which the main lobe splits. An examination of the derivative $d E_{\phi} / d \phi$ as $\phi \rightarrow 0^{\circ}$ is necessary to determine when the main lobe begins to split into two lobes. Lobe-splitting occurs when

$$
\lim _{\phi \rightarrow 0} \frac{d E_{\phi}}{d \phi}=-4 \sin \left[\frac{\pi D}{\lambda}\left(1-\cos \phi_{0}\right)\right]\left\{\frac{2 \pi D}{\lambda}+\sin \left[\frac{\pi D}{\lambda}\left(1-\cos \phi_{0}\right)\right]\right\}
$$

is positive. Lobe-splitting takes place when $\sin \left[\pi D / \lambda\left(1-\cos \phi_{0}\right)\right]$ is negative, since $2 \pi D / \lambda+$ $\sin \left[\pi D / \lambda\left(1-\cos \phi_{0}\right)\right]$ is always positive. Sin $\left[\pi D / \lambda\left(1-\cos \phi_{0}\right)\right]$ is negative when $\pi D / \lambda$ $\left(1-\cos \phi_{0}\right)>\pi$; therefore, lobe-splitting occurs when 
FIgURE 12. Two- and three-loop array beamwidth comparison.

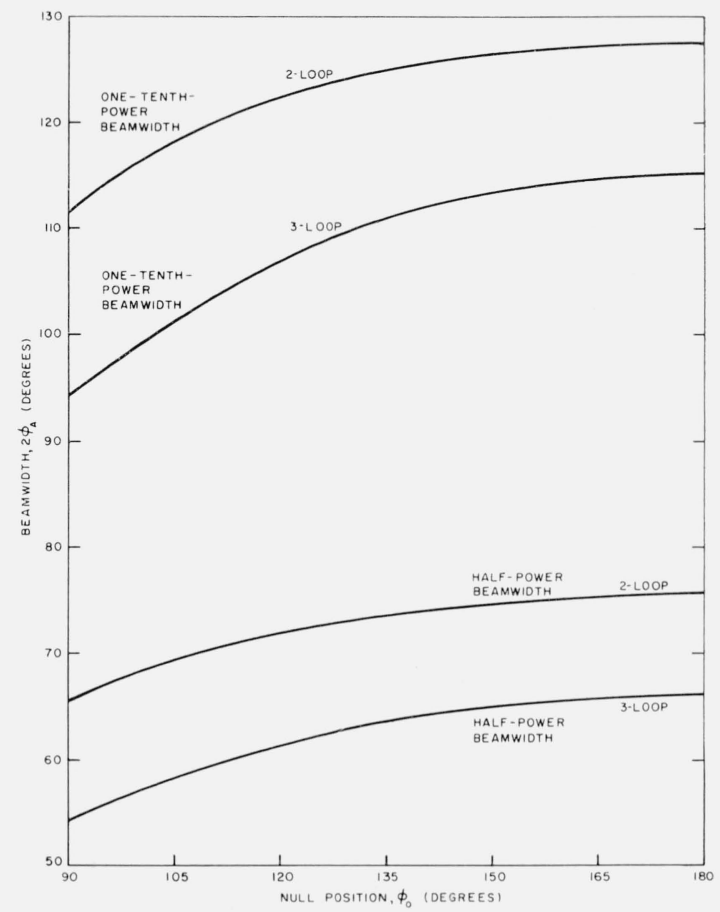

$$
\frac{D}{\lambda}>\frac{1}{1-\cos \phi_{0}} .
$$

By inspection of (29) it must be concluded that there is no lobe-splitting for loop spacings below a half wavelength.

\section{d. Front-to-Back Lobe Ratios}

Another important aspect of the directivity of the three-loop array is the ratio of the maximum of the front lobe to the maximum of the back or side lobes, which shows the extent to which signals from the rear $\left(\phi=90\right.$ to $\left.270^{\circ}\right)$ are excluded. These ratios are derived from (24). If the amplitude of the front lobe is divided by the amplitude of the side lobes, the ratio is

$$
\frac{E_{\phi=0^{\circ}}}{E_{\phi=\phi_{1}}}=R_{1}=\frac{\sin ^{2}\left[\frac{\pi D}{\lambda}\left(1-\cos \phi_{0}\right)\right]}{\left(\frac{2 \pi D}{\lambda}\right)^{2} \cos ^{3} \phi_{1}} .
$$

Similarly, the ratio of the front lobe to the back lobe is

$$
\frac{E_{\phi=0^{\circ}}}{E_{\phi=180^{\circ}}}=R_{0}=\frac{-\sin ^{2}\left[\frac{\pi D}{\lambda}\left(1-\cos \phi_{0}\right)\right]}{\sin ^{2}\left[\frac{\pi D}{\lambda}\left(1+\cos \phi_{0}\right)\right]} .
$$

These ratios are shown as a function of loop spacing in figures 13 and 14 for a variety of null positions. In all cases the maximum front-to-back ratio or greatest directivity occurs at the closest loop spacing. This is contrary to the usual array in which directivity increases with aperture length. This again shows superdirectivity tendencies. 

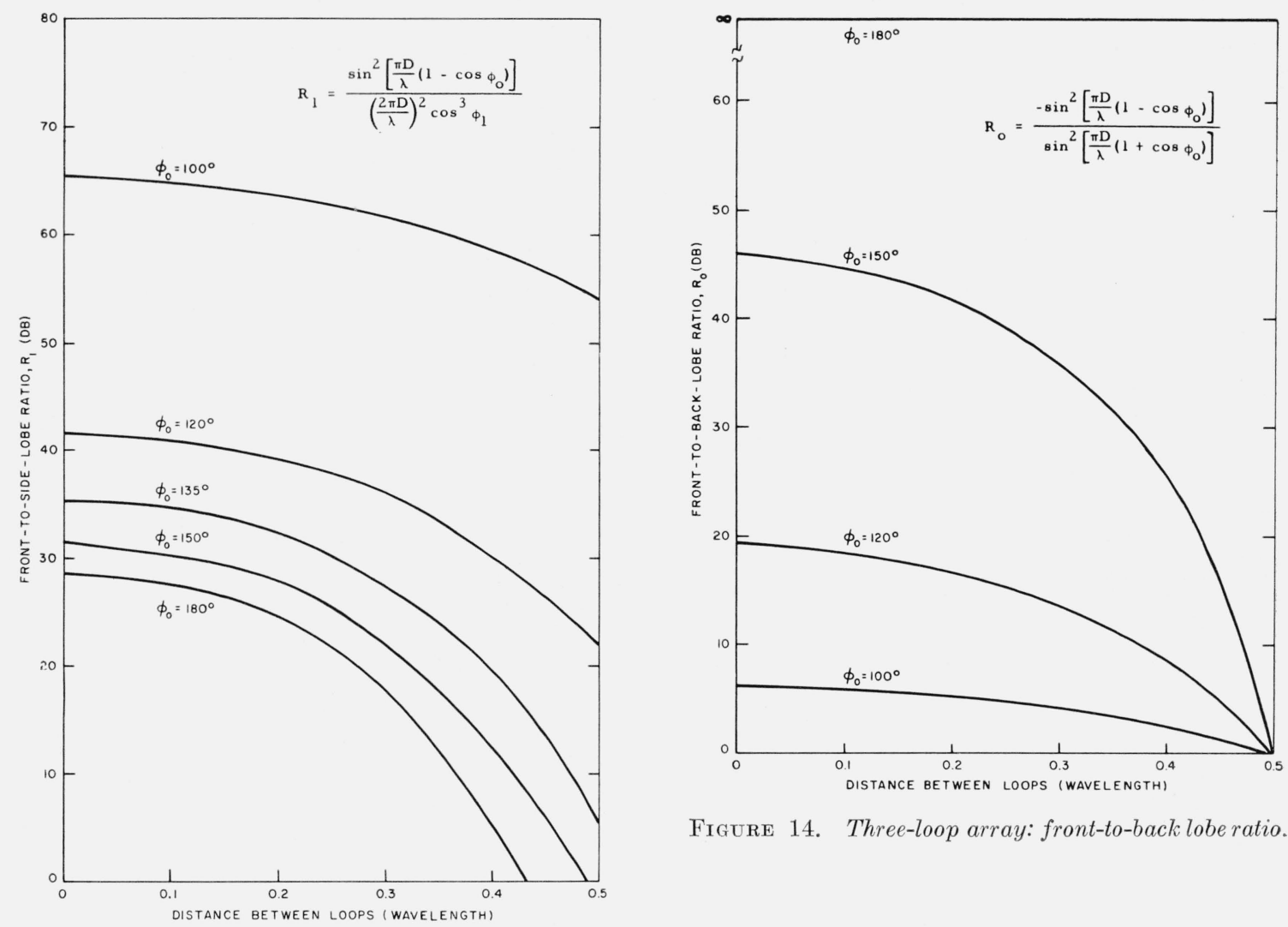

FiguRE 14. Three-loop array: front-to-back lobe ratio.

FIGURE 13. Three-loop array: front-to-side lobe ratio.

For small loop spacing, (30) and (31) reduce to

$$
R_{1}=\frac{27}{4} \frac{\left(1-\cos \phi_{0}\right)^{2}}{\cos ^{3} \phi_{0}}
$$

and

$$
R_{0}=-\left(\frac{1-\cos \phi_{0}}{1+\cos \phi_{0}}\right)^{2}
$$

If $R_{1}$ and $R_{0}$ are plotted as a function of the null position, as shown in figure 15, an optimum null position is apparent where $R_{1}$ equals $R_{0}$. This condition occurs at $\phi_{0}=138.6$ and $221.4^{\circ}$, where $R_{1}$ and $R_{0}$ are 49 (or $33.8 \mathrm{db}$ ). A very wide range of null positions can be selected in which the front-to-back ratio is greater than $28 \mathrm{db}$.

In applications where the three-loop array is in a multiple-source field, such as sferics at VLF, it is important to optimize the ratio of power collected by the front lobe to that collected by the back lobes. The power ratio may be derived from (24). For small loop spacings, (24) reduces to

$$
\left|E_{\phi}\right|=\cos \phi\left[\frac{2 \pi D}{\lambda}\left(\cos \phi-\cos \phi_{0}\right)\right]^{2}
$$

The ratio of power in the front lobe to that in the back lobe is

$$
R_{p}=\frac{P_{f}}{P_{b}}=\frac{\int_{0}^{\frac{\pi}{2}}\left|E_{\phi}\right|^{2} d \phi}{\int_{\frac{\pi}{2}}^{\pi}\left|E_{\phi}\right|^{2} d \phi},
$$




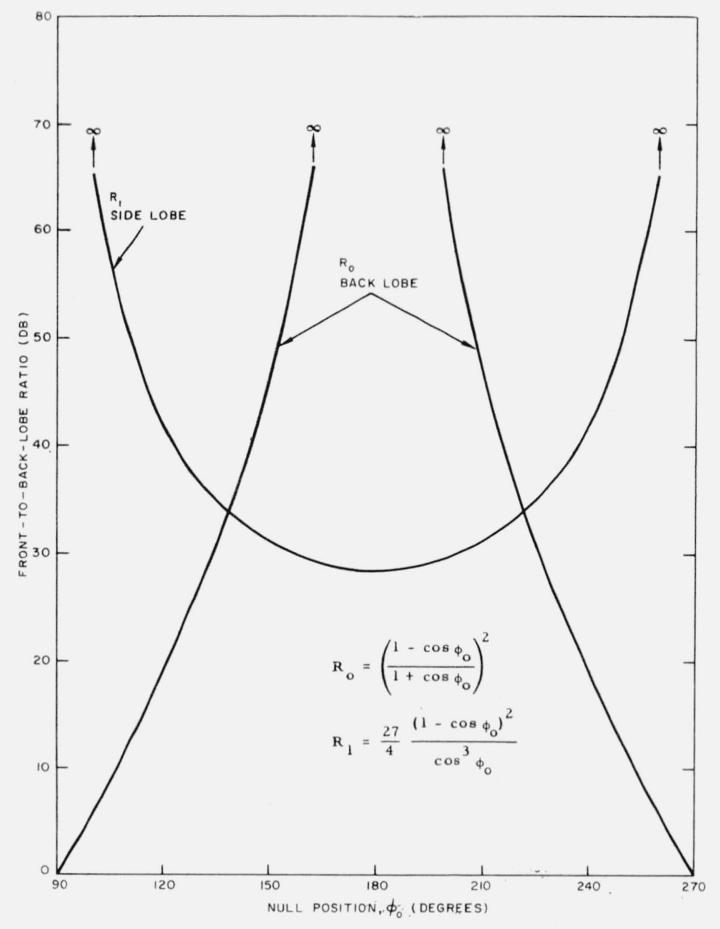

Figure 15. Three-loop array: front to back and front to side lobe ratio for small-loop spacing.

The power need only be integrated over half the pattern since there is symmetry about $\phi=0^{\circ}$.

$$
R_{p}=\frac{P_{f} / 2}{P_{b} / 2}=\frac{32\left(\frac{\pi D}{\lambda}\right)^{4}\left(\frac{\pi}{4} \cos ^{4} \phi_{0}-\frac{8}{3} \cos ^{3} \phi_{0}+\frac{9}{8} \pi \cos ^{2} \phi_{0}-\frac{32}{15} \cos \phi_{0}+\frac{15}{96} \pi\right)}{32\left(\frac{\pi D}{\lambda}\right)^{4}\left(\frac{\pi}{4} \cos ^{4} \phi_{0}+\frac{8}{3} \cos ^{3} \phi_{0}+\frac{9}{8} \pi \cos ^{2} \phi_{0}+\frac{32}{15} \cos \phi_{0}+\frac{15}{96} \pi\right)},
$$

which reduces to

$$
R_{p}=\frac{\pi\left(\frac{1}{4} \cos ^{4} \phi_{0}+\frac{9}{8} \cos ^{2} \phi_{0}+\frac{15}{96}\right)-\left(\frac{8}{3} \cos ^{3} \phi_{0}+\frac{32}{15} \cos \phi_{0}\right)}{\pi\left(\frac{1}{4} \cos ^{4} \phi_{0}+\frac{9}{8} \cos ^{2} \phi_{0}+\frac{15}{96}\right)+\left(\frac{8}{3} \cos ^{3} \phi_{0}+\frac{32}{15} \cos \phi_{0}\right)}
$$

The front-to-back power ratio depends only upon the position of the null when loop spacing is small. Equation (37), which is plotted in figure 16 shows that the power ratio remains above $30 \mathrm{db}$ over a wide range of null positions. The optimum front-to-back power ratio of $35 \mathrm{dh}$ occurs at 144 and $216^{\circ}$.

\section{Limiting Factors in Loop Spacing}

Although the results show that the smaller the loop spacing the greater the directivity, there are certain limitations. The limiting factors in loop spacing are the amplitude and phase inequality in the loop voltages received in the direction of a null and to a lesser extent the coupling between loops. Coupled with this is the decrease in amplitude of the main lobe of the array as the loops are spaced closer.

\subsection{Amplitude and Phase Inequality}

The resultant null voltage from a pair of loops is derived in appendix 5.2, and is expressed as 


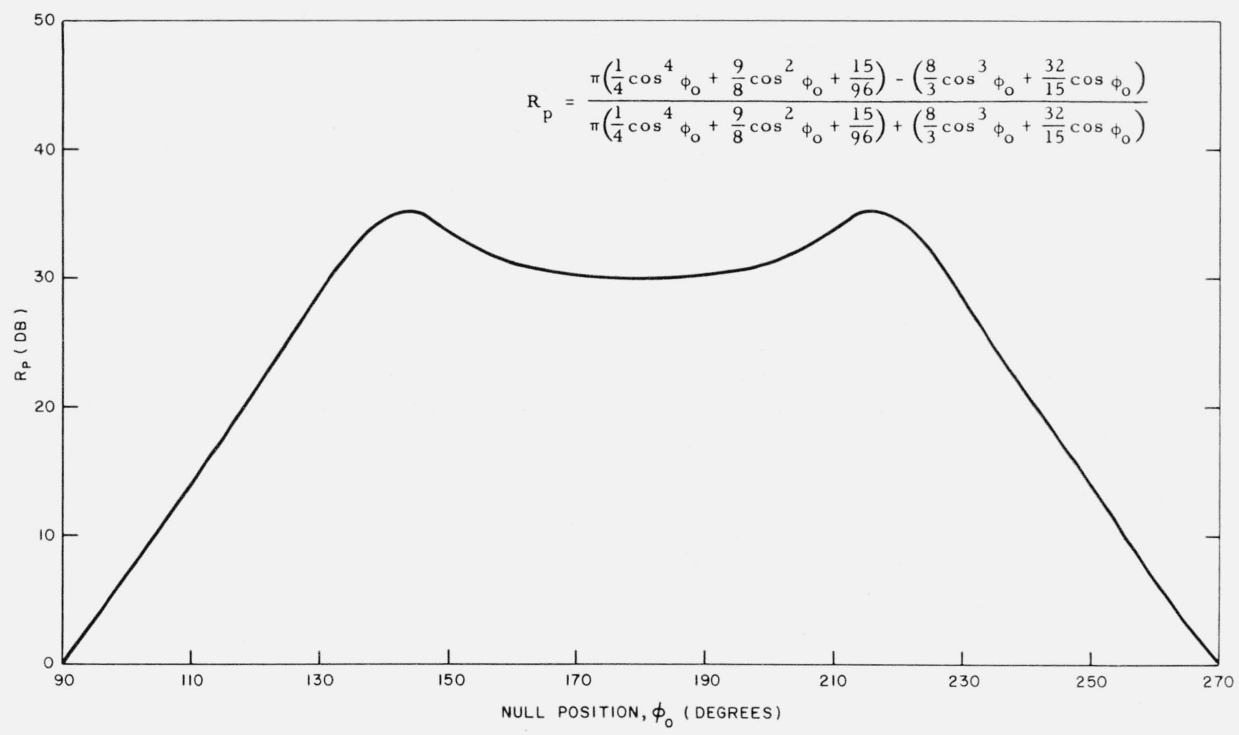

FiguRE 16. Three-loop array: ratio of power in front lobe to power in back lobe.

$$
\left|e_{\tau}\right|=\Delta e_{12}-j \Delta \theta_{12} E_{1}
$$

where $\Delta e_{12}$ is the amplitude difference between the loop voltages and $\Delta \theta_{12}$ is the phase difference between the loop voltages; $E_{1}$ is the voltage from one loop.

In a similar manner the resultant null voltage from a three-loop array derived in appendix 5.2 is

where

$$
\left|e_{r}\right|=\Delta e_{12}+\Delta e_{23}-j\left(\Delta \theta_{1} E_{1}+\Delta \theta_{3} E_{3}\right),
$$

$\Delta e_{23}$ is the amplitude difference between loop 2 and 3 , $\Delta \theta_{23}$ is the phase difference between loop 2 and 3 , and $E_{3}$ is the voltage from loop 3.

The resultant null voltage can become quite large and obscure the null if the loop voltages are unequal and out of phase. The amplitude difference is most important because the phase difference tends to simply relocate the null position, while the amplitude difference limits the depth of the null. This is especially true in the two-loop array case. In the three-loop array case, phase difference between the loop voltages tends to create two null positions. One percent difference in amplitude and $1^{\circ}$ phase shift between the voltages will produce a null voltage of $0.01 E_{L}$ in the case of the two-loop array and a maximum null voltage of $0.04 E_{L}$ in the case of the three-loop array.

\subsection{Coupling Between Loops}

Coupling between loops could be a serious problem in loop arrays if sufficient voltage is coupled from one loop to another to cause an inequality in the loop voltages. The coupling between adjacent loops may be determined by deriving the ratio of the field produced by the adjacent loop to the free space radiation field. These ratios for the $E$ and $H$ fields derived in appendix 5.3 are 


$$
\frac{E_{\theta}}{E_{f}}=\frac{1}{\ln \frac{r}{r_{0}}}\left[\frac{\pi^{2}}{2}\left(\frac{r}{D}\right)^{2}\left(\frac{r}{\lambda}\right)+j \pi^{3}\left(\frac{r}{D}\right)\left(\frac{r}{\lambda}\right)^{2}\right] /-\frac{2 \pi D}{\lambda}
$$

and

$$
\frac{H_{\phi}}{H_{f}}=-\frac{1}{\ln \frac{r}{r_{0}}}\left[\frac{\pi^{2}}{2}\left(\frac{r}{D}\right)^{2}\left(\frac{r}{\lambda}\right)+j \pi^{3}\left(\frac{r}{D}\right)\left(\frac{r}{\lambda}\right)^{2}-j \frac{\pi}{4}\left(\frac{r}{D}\right)^{3}\right] /-\frac{2 \pi D}{\lambda} .
$$

From (40) and (41) it is obvious that the ratio of the fields will be quite small if the ratios of loop radius to loop spacing and loop radius to wavelength are small.

At VLF frequencies the loop radius of a practical array is by necessity small compared to the wavelength, and the loop radius can be made small compared to loop spacing so the ratio of the fields and hence the coupled voltage will be quite small. For example, if

$$
r / \lambda=0.0001, r / D=0.01 \text { and } r / r_{0}=0.1 \text {. }
$$

Then the ratio of fields or ratio of coupled voltage to received voltage is

$$
E_{\theta} / E_{f}=2 \times 10^{-8}+j 0.15 \times 10^{-8} \text { and } H_{\phi} / H_{f}=-j 70 \times 10^{-8}
$$

which is very small and insignificant.

\subsection{Front Lobe Amplitude}

The other limitation on loop proximity is the amplitude of the front lobe. For the threeloop array (24) gives

$$
E_{\phi=0^{\circ}}=4 \sin ^{2}\left[\frac{\pi D}{\lambda}\left(1-\cos \phi_{0}\right)\right]
$$

which is the amplitude of the front lobe normalized to the voltage from one loop. For very close loop spacings the amplitude of the front lobe is quite small. The amplitude is equal to the voltage from one loop when the spacing is 0.0833 wavelength and decreases rapidly as the loops are brought closer together. When the loops are spaced at less than 0.1 wavelength, (42) reduces to

$$
E_{\phi=0^{\circ}}=\left[\frac{2 \pi D}{\lambda}\left(1-\cos \phi_{0}\right)\right]^{2}
$$

For the optimum array (that is, equal back lobes) (43) reduces to

$$
E_{\phi=0^{\circ}}=121\left(\frac{D}{\lambda}\right)^{2}
$$

Similarly for a two-loop array with equal back lobes and close loop spacing the front lobe amplitude is

$$
E_{\phi=0^{\circ}}=11.4 D / \lambda \text {. }
$$

The limitation on loop spacing is readily apparent if the front lobe amplitudes (44) and (45) are compared to the null voltages with 1 percent difference and $1^{\circ}$ phase shift in loop voltages. In the case of the two-loop array the null voltage and front lobe are equal at a loop spacing of 0.0017 wavelength. The three-loop array has equal null and front lobe voltage at a loop spacing of 0.018 wavelength. Of course the back lobes will be obscured at even greater loop spacings.

\section{Conclusions}

The three-loop array has greater directivity than the two-loop array, and the directivity is greatest for small loop spacing. The beamwidth is narrowest, the front-to-back voltage and power ratios are greatest, and the position of the back lobes and nulls are most invariant 
when closely spaced loops are used. Another important feature of the loop array-particularly useful for receiving sferics - is its broad bandwidth. Its characteristics change very little with frequency for close loop spacings since there is very little coupling between loops.

Any inequality in the signals from the loops tends to obscure the front and back lobes; this will limit the proximity of the loops.

A summary of the derived equations is presented in tables 1 and 2 .

TABLE 1. Summary of equations for the three-loop array

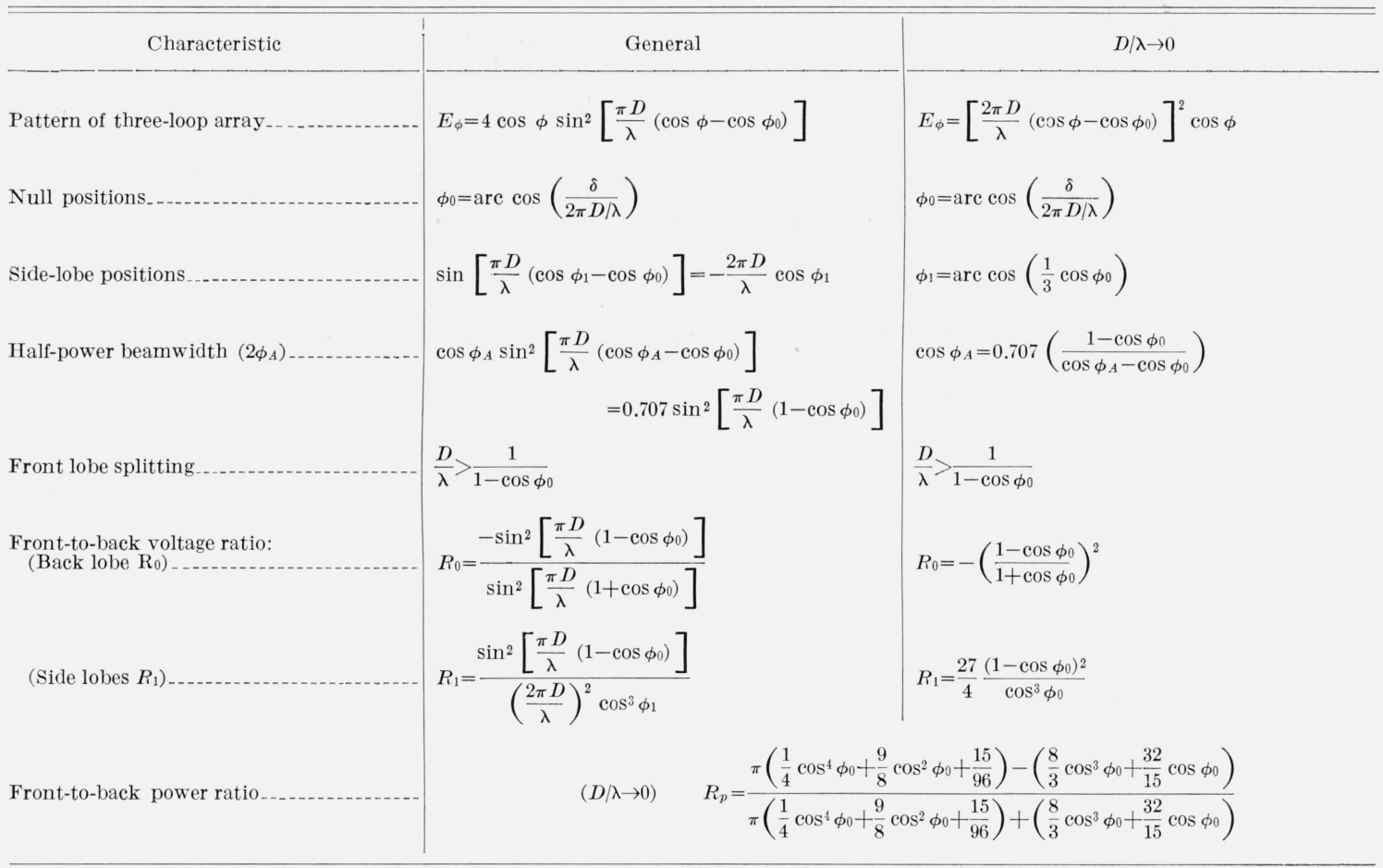

TABLE 2. Summary of equations for the two-loop array

\begin{tabular}{|c|c|c|}
\hline Characteristic & General & $D / \lambda \rightarrow 0$ \\
\hline Pattern of two-loop array ..- & $E_{\phi}=2 \cos \phi \sin \left[\frac{\pi D}{\lambda}\left(\cos \phi-\cos \phi_{0}\right)\right]$ & $E_{\phi}=2 \pi D / \lambda\left(\cos ^{2} \phi-\cos \phi \cos \phi_{0}\right)$ \\
\hline Null positions_... & $\phi_{0}=\arccos \left[\frac{\delta}{2 \pi D / \lambda}\right]$ & $\phi_{0}=\arccos \left(\frac{\delta}{2 \pi D / \lambda}\right)$ \\
\hline Side-lobe positions & $\tan \left[\frac{\pi D}{\lambda}\left(\cos \phi_{1}-\cos \phi_{0}\right)\right]=\frac{-\pi D}{\lambda} \cos \phi_{1}$ & $2 \cos \phi_{1}=\cos \phi_{0}$ \\
\hline Half-power beamwidth $\left(2 \phi_{A}\right) \ldots$ & $\begin{aligned} \cos \phi_{A} \sin \left[\frac{\pi D}{\lambda}\left(\cos \phi_{0}\right)\right] & \\
& =0.707 \sin \left[\frac{\pi D}{\lambda}\left(1-\cos \phi_{0}\right)\right.\end{aligned}$ & $\begin{array}{l}\cos \phi_{A}=\frac{1}{2} \cos \phi_{0} \pm \frac{1}{2} \\
\sqrt{\cos ^{2} \phi_{0}-2.82\left(\cos \phi_{0}-1\right)}\end{array}$ \\
\hline $\begin{array}{l}\text { Front-to-back voltage ratio: } \\
\quad \text { (Side lobes } R_{1} \text { ) }\end{array}$ & $R_{1}=\frac{\sin \left[\pi D / \lambda\left(1-\cos \phi_{0}\right)\right]}{\cos \phi_{1} \sin \left[\pi D / \lambda\left(\cos \phi_{1}-\cos \phi_{0}\right)\right]}$ & $R_{1}=\frac{1-\cos \phi_{0}}{\cos \phi_{1}\left(\cos \phi_{1}-\cos \phi_{0}\right)}$ \\
\hline (Back lobe $\left.R_{0}\right)_{\ldots}$ & $R_{0}=\frac{\sin \left[\frac{\pi D}{\lambda}\left(1-\cos \phi_{0}\right)\right]}{\sin \left[\frac{\pi D}{\lambda}\left(1+\cos \phi_{0}\right)\right]}$ & $R_{0}=\frac{1-\cos \phi_{0}}{1+\cos \phi_{0}}$ \\
\hline $\begin{array}{l}\text { Ratio of power in the front lobe to that in } \\
\text { the back lobes. }\end{array}$ & $R_{p}=\frac{\pi}{\pi}$ & 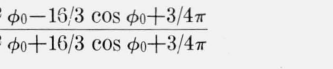 \\
\hline
\end{tabular}


The author thanks Vernon Kamp and Ivan Rainwater for their effort in calculating the curves in the figures.

\section{Appendix}

\subsection{Loop Array Received Voltage Analysis}

If the loops are oriented in line in a vertical plane and receiving vertically polarized energy equally in all loops from a linear wave front, the resultant voltage received at an angle $\phi$ in the horizontal plane can be derived with the aid of figure A1.

If the receiving elements in figure $A 1$ are first considered to be isotropies with delay, $-\delta$, as shown

Factoring out $e^{-j \delta}$

$$
E_{\phi}=e^{-j \delta}+e^{-j \delta}-e^{-j(\psi+2 \delta)}-e^{j \psi} .
$$

$$
E_{\phi}=e^{-j \delta}\left[1+1-e^{-j(\psi-\delta)}-e^{j(\psi+\delta)}\right] .
$$

By deMoivre's theorem

$$
\begin{gathered}
e^{j x}+e^{-j x}=2 \cos x \\
E_{\phi}=2[1-\cos (\psi+\delta)] /-\delta .
\end{gathered}
$$

Three-Loop Array

By pattern multiplication [Kraus, 1950a] the received voltage of the three-loop array is

$$
E_{\phi}=2 \cos \phi[1-\cos (\psi+\delta)] \underline{/-\delta} .
$$

From inspection of $(5 \mathrm{~A})$ the null positions occur when

$$
\cos (\psi+\delta)=1
$$

or

$$
\psi+\delta=0,2 \pi, 4 \pi, \ldots ;
$$

then

$$
\psi=-\delta
$$

If

$$
\psi=\frac{2 \pi D}{\lambda} \cos \phi
$$

then

$$
-\delta=\frac{2 \pi D}{\lambda} \cos \phi_{0}
$$

where $\phi_{0}$ is the null position. Then substituting (10A) into (5A) the result is

Figure A1.

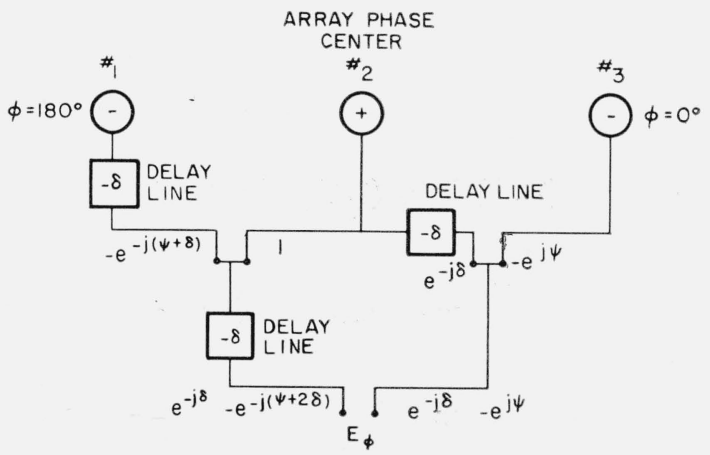




$$
\begin{aligned}
E_{\phi} & =2 \cos \phi\left[1-\cos \left(\frac{2 \pi D}{\lambda} \cos \phi-\frac{2 \pi}{\lambda} \cos \phi_{0}\right)\right] \underline{1-\delta} \\
& =2 \cos \phi\left[1-\cos \left[\frac{2 \pi D}{\lambda}\left(\cos \phi-\cos \phi_{0}\right)\right]\right] /-\delta .
\end{aligned}
$$

By using the trigonometric identity

$$
1-\cos 2 x=2 \sin ^{2} x
$$

the received voltage reduces to

$$
\begin{gathered}
E_{\phi}=4 \cos \phi \sin ^{2}\left[\frac{\pi D}{\lambda}\left(\cos \phi-\cos \phi_{0}\right)\right] \underline{1-\delta} . \\
\text { Two-Loop Array }
\end{gathered}
$$

If only the 1 st and $2 \mathrm{~d}$ receiving elements are used in figure $\mathrm{A} 1$

$$
E_{\phi}=1-e^{-j(\psi+\delta)} .
$$

Factoring $e^{-j\left(\frac{\psi}{2}+\frac{\delta}{2}\right)}$ out of $(14 \mathrm{~A})$ we have

$$
E_{\phi}=e^{-j\left(\frac{\psi}{2}+\frac{\delta}{2}\right)}\left[e^{+j\left(\frac{\psi}{2}+\frac{\delta}{2}\right)}-e^{-j\left(\frac{\psi}{2}+\frac{\delta}{2}\right)}\right] .
$$

By deMoivre's theorem

$$
\begin{gathered}
e^{j x}-e^{-j x}=2 \sin x \\
E_{\phi}=\left[2 \sin \left(\frac{\psi}{2}+\frac{\delta}{2}\right)\right]-\frac{\psi}{2}-\frac{\delta}{2}
\end{gathered}
$$

By pattern multiplication [Kraus 1950a] the received voltage of the two loop array is

$$
E_{\phi}=\left[2 \cos \phi \sin \left(\frac{\psi}{2}+\frac{\delta}{2}\right)\right] /-\left(\frac{\psi}{2}+\frac{\delta}{2}\right) .
$$

From inspection of (18A) the null positions occur when

$$
\sin \left(\frac{\psi}{2}+\frac{\delta}{2}\right)=0
$$

or

$$
\psi+\delta=0,2 \pi, 4 \pi, \ldots .
$$

This is the same as the three-loop case. Then

$$
\psi=-\delta
$$

since

$$
\psi=\frac{2 \pi D}{\lambda} \cos \phi
$$

then

$$
-\delta=\frac{2 \pi D}{\lambda} \cos \phi_{0} \text { where } \phi_{0} \text { is the null position. }
$$

Substituting (22A) and (23A) into (18A) the two-loop received voltage is

$$
\begin{aligned}
E_{\phi} & =2 \cos \phi\left[\sin \left(\frac{\pi D}{\lambda} \cos \phi-\frac{\pi D}{\lambda} \cos \phi_{0}\right)\right] /-\frac{\psi}{2}-\frac{\delta}{2} \\
& =2 \cos \phi \sin \left[\frac{\pi D}{\lambda}\left(\cos \phi-\cos \phi_{0}\right)\right]-\left(\frac{\psi+\delta}{2}\right) .
\end{aligned}
$$




\subsection{Null Voltage Analysis}

The null voltage will depend on the equality and phase difference between the individual received loop voltages. The effect of amplitude and phase differences between loop voltages on the resultant null voltage can be determined using figure A2. If the loop received voltages are assumed to be sinusoidal they may be added to show the effect of small phase differences, $\Delta \theta$, and amplitude difference on the resultant null voltage, $E_{r}$. When only loops No. 1 and No. 2 are considered, the null voltage of a two-loop array is

$$
e_{r}=E_{2} \sin \omega t-E_{1} \sin \left(\omega t \pm \Delta \theta_{1}\right) .
$$

By using the trigonometric identity

$$
\begin{gathered}
\sin (x \pm y)=\sin x \cos y \pm \cos x \sin y \\
e_{r}=E_{2} \sin \omega t-E_{1}\left[\sin \omega t \cos \left( \pm \Delta \theta_{1}\right) \pm \cos \omega t \sin \pm \theta_{1}\right]
\end{gathered}
$$

when $\Delta \theta_{1}$ is small

$$
\sin \pm \Delta \theta \approx \pm \Delta \theta \text { and } \cos \pm \Delta \theta_{1} \approx 1 .
$$

Using these approximations,

$$
\begin{aligned}
e_{r} & =E_{2} \sin \omega t-E_{1} \sin \omega t-\Delta \theta_{1} E_{1} \cos \omega t \\
& =\left(E_{2}-E_{1}\right) \sin \omega t-\Delta \theta_{1} E_{1} \cos \omega t \\
& =\Delta e_{12} \sin \omega t-\Delta \theta_{1} E_{1} \cos \omega t
\end{aligned}
$$

where

The amplitude of

$$
\Delta e_{12}=E_{2}-E_{1}
$$

$$
\left|e_{r}\right|=E_{r}=\left|\Delta e_{12}-j \Delta \theta_{1} E_{1}\right| .
$$

The null voltage of the three-loop array can be derived similarly by summing all three loop voltages shown in figure A2.

By using 26A,

$$
e_{r}=2 E_{2} \sin \omega t-E_{1} \sin \left(\omega t \pm \Delta \theta_{12}\right)-E_{3} \sin \left(\omega t \pm \Delta \theta_{23}\right) .
$$

$$
\begin{array}{r}
e_{r}=2 E_{2} \sin \omega t-E_{1}\left[\sin \omega t \cos \pm \Delta \theta_{12} \pm \cos \omega t \sin \pm \Delta \theta_{12}\right] \\
-E_{3}\left[\sin \omega t \cos \pm \Delta \theta_{23} \pm \cos \omega t \sin \pm \Delta \theta_{23}\right]
\end{array}
$$

Figure A2.

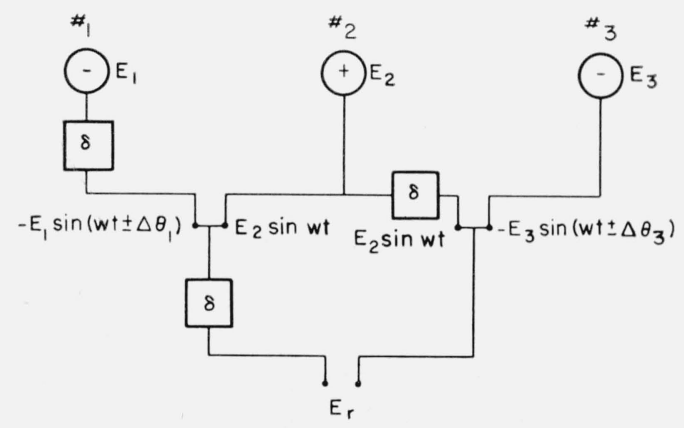


When $\Delta \theta_{12}$ and $\Delta \theta_{23}$ are small

T'hen

$$
\begin{gathered}
\sin \pm \Delta \theta_{12} \approx \pm \Delta \theta_{12} \\
\sin \pm \theta_{23} \approx \pm \Delta \theta_{23} \\
\cos \pm \theta_{12} \approx 1 \\
\cos \pm \theta_{23} \approx 1
\end{gathered}
$$

$$
\begin{aligned}
e_{r} & =2 E_{2} \sin \omega t-E_{1}\left[\sin \omega t+\Delta \theta_{12} \cos \omega t\right]-E_{3}\left[\sin \omega t+\Delta \theta_{23} \cos \omega t\right] \\
& =2 E_{2} \sin \omega t-E_{1} \sin \omega t-E_{3} \sin \omega t-E_{1} \Delta \theta_{12} \cos \omega t-E_{3} \Delta \theta_{23} \cos \omega t \\
& =\left[\left(E_{2}-E_{1}\right)+\left(E_{2}-E_{3}\right)\right] \sin \omega t-\left(E_{1} \Delta \theta_{1}+E_{3} \Delta \theta_{3}\right) \cos \omega t .
\end{aligned}
$$

From (40A) it is evident that the amplitude of $E_{r}$ is

$$
E_{r}=\left|\Delta e_{12}+\Delta e_{23}-j\left(E_{1} \Delta \theta_{12}+E_{3} \Delta \theta_{23}\right)\right| \text {. }
$$

\subsection{Coupling Between Loops}

The voltage induced in a loop by a nearby loop may be an appreciable fraction of the voltage induced in that same loop by the free-space radiation field. The coupling between adjacent loops can be determined by deriving the ratio of the field produced by the adjacent loop to the free-space radiation field.

If two small single-turn loops are oriented in line in the $\theta$-plane, the field intensity produced by one loop in the vicinity of the other is given by [Schelkunoff, 1952]

$$
\begin{gathered}
E_{\theta}=\frac{\eta \beta^{2} I A}{4 \pi D}\left(1+\frac{1}{j \beta D}\right) e^{-j \beta D} \cos \phi \\
H_{\phi}=\frac{\beta^{2} I A}{4 \pi D}\left(1+\frac{1}{j \beta D}-\frac{1}{\beta^{2} D^{2}}\right) e^{-j \beta D} \cos \phi .
\end{gathered}
$$

In the plane of the loops

$$
\begin{gathered}
E_{\theta}=\frac{\beta^{2} I A}{4 \pi D}\left(1+\frac{1}{j \beta D}\right) /-\beta D \\
H_{\phi}=-\frac{\beta^{2} I A}{4 \pi D}\left(1+\frac{1}{j \beta D}-\frac{1}{\beta^{2} D^{2}}\right) /-\beta D .
\end{gathered}
$$

Equations (44A) and (45A) are the field components in the vicinity of the loop produced by current $I$ in the nearby loop. If the current induced into the nearby loop were known the ratio of the coupled field to the free-space field could be derived. The maximum voltage induced in the nearby loop antenna in a free space radiation field is [Jean, Taggart, and Wait, 1961].

from which

$$
\begin{gathered}
V_{L}=j \beta A E_{f} \\
I=\frac{V_{L}}{Z_{L}}=j \frac{\beta A E_{f}}{Z_{L}} .
\end{gathered}
$$

Substituting (47A) into (44A) and (45A) with $\beta=\frac{2 \pi}{\lambda}$ and $\mathrm{A}=\pi r^{2}$

$$
\frac{E_{\theta}}{E_{f}}=\left(\frac{\eta}{Z_{L}}\right) \frac{\pi^{3} r^{4}}{D \lambda^{2}}\left(\frac{1}{D}-j \frac{2 \pi}{\lambda}\right) \underline{/-\beta D}
$$

and similarly for the magnetic field, with $\frac{E_{f}}{H_{f}}=\eta$, 


$$
\frac{H_{\phi}}{H_{f}}=-\left(\frac{\eta}{Z_{L}}\right) \frac{\pi^{3} r^{4}}{D \lambda^{2}}\left(\frac{1}{D}+j \frac{2 \pi}{\lambda}-j \frac{1}{2 \pi D^{2} / \lambda}\right) /-\frac{2 \pi}{\lambda} D
$$

If $(48 \mathrm{~A})$ and $(49 \mathrm{~A})$ are rearranged in terms of the ratios of loop radius to loop separation and loop radius to wavelength,

$$
\frac{E_{\theta}}{E_{f}}=\left(\frac{\eta}{Z_{L}}\right)\left[\pi^{3}\left(\frac{r}{D}\right)^{2}\left(\frac{r}{\lambda}\right)^{2}+j 2 \pi^{4}\left(\frac{r}{D}\right)\left(\frac{r}{\lambda}\right)^{3}\right] /-2 \pi \frac{D}{\lambda}
$$

and

$$
\frac{H_{\phi}}{H_{f}}=-\frac{\eta}{Z_{L}}\left[\pi^{3}\left(\frac{r}{D}\right)^{2}\left(\frac{r}{\lambda}\right)^{2}+2 \pi^{4}\left(\frac{r}{D}\right)\left(\frac{r}{\lambda}\right)^{3}-j \frac{\pi^{2}}{2}\left(\frac{r}{D}\right)^{3}\left(\frac{r}{\lambda}\right)\right] /-2 \pi \frac{D}{\lambda}
$$

For small loops the impedance is [Schelkunoff, 1952]

$$
\begin{gathered}
Z_{L}=240 \pi^{2} \frac{r}{\lambda} \ln \frac{r}{r_{0}} \\
\frac{\eta}{Z_{L}}=\frac{120 \pi}{240 \pi^{2} \frac{r}{\lambda} \ln \frac{r}{r_{0}}}=\frac{1}{2 \pi \frac{r}{\lambda} \ln \frac{r}{r_{0}}}
\end{gathered}
$$

Substituting (53A) into (50A) and (51A)

and

$$
\frac{E_{\theta}}{E_{f}}=\frac{1}{\ln \frac{r}{r_{0}}}\left[\frac{\pi^{2}}{2}\left(\frac{r}{D}\right)^{2}\left(\frac{r}{\lambda}\right)+j \pi^{3}\left(\frac{r}{D}\right)\left(\frac{r}{\lambda}\right)^{2}\right] /-2 \pi \frac{D}{\lambda}
$$

$$
\frac{H_{\phi}}{H_{f}}=-\frac{1}{\ln \frac{r}{r_{0}}}\left[\frac{\pi^{2}}{2}\left(\frac{r}{D}\right)^{2}\left(\frac{r}{\lambda}\right)+j \pi^{3}\left(\frac{r}{D}\right)\left(\frac{r}{\lambda}\right)^{2}-j \frac{\pi}{4}\left(\frac{r}{D}\right)^{3}\right] / \underline{-\frac{2 \pi D}{\lambda}}
$$

The ratio of the fields would be quite small for practical antennas at VLF frequencies, for example if $r / \lambda=10^{-4}$ and $r / D=0.01$ and $r / r_{o}=.1$

$$
\frac{E_{\theta}}{E_{f}}=2 \times 10^{-8}+j 0.15 \times 10^{-8} \text { and } \frac{H_{\phi}}{H_{f}}=-j 70 \times 10^{-8} .
$$

\section{References}

Friis, H. T. (December 1925), a new directional receiving system, Proc. IRE 13, 685-707.

Jean, A. G., H. E. Taggart, and J. R. Wait (July-Sept. 1961), Calibration of loop antennas at VLF, J. Res.

NBS 65C (Eng. and Instr.), No. 3, 189-193.

Kraus, J. D. (1950), Antennas, (McGraw Hill Book Co. Inc., New York).

Riblet, H. J. (May 1948), Note on the maximum directivity of an antenna, Proc. IRE 36, 620-624.

Schelkunoff, S. A. and H. T. Friis (1952), Antenna theory and practice, (John Wiley \& Sons, Inc., New York, N.Y.).

Stearns, C. O. (To be published), Computed performance of moderate size super-gain antennas.

Taylor, T. T. (Sept. 1948), A discussion of the maximum directivity of an antenna, Proc. IRE 36, 1135.

Toraldo di Francia, G. (July 1956), Directivity, super-gain and information, IRE, Trans. Antennas Propagation AP-4, 473-479.

Wilmotte, R. M. (July 1948), Note on practical limitations in the directivity of antennas, Proc. IRE $36,878$.

Yaru, N. (Sept. 1951), A note on super-gain antenna arrays, Proc. IRE 39, 1081-1085. 